\title{
Olanzapine and fluoxetine combination therapy for treatment-resistant depression: review of efficacy, safety, and study design issues
}

This article was published in the following Dove Press journal:

Neuropsychiatric Disease and Treatment

I July 2009

Number of times this article has been viewed

\author{
William $\vee$ Bobo \\ Richard C Shelton \\ Department of Psychiatry, Vanderbilt \\ University School of Medicine, \\ Nashville, TN, USA
}

\begin{abstract}
Treatment-resistant depression (TRD) is a common occurrence in clinical practice. Up to $30 \%$ of patients with major depression do not respond to conventional antidepressant treatment, while a significantly greater number of patients experience only partial symptom reduction. Numerous strategies may be applied by the practicing clinician to overcome limitations in the effectiveness of antidepressant monotherapy, including combining drug treatment with evidence-supported psychotherapies, combining antidepressants (combination pharmacotherapy), and combining antidepressants with other non-antidepressant psychotropic medications (augmentation treatment). One such augmentation strategy, the combination of the selective serotonin reuptake inhibitor, fluoxetine (FLX), with the atypical antipsychotic drug, olanzapine (OLZ), is supported by the results of four randomized, double-blind, acute phase studies of patients who had responded inadequately to antidepressant monotherapy. In each study, the FLX/OLZ combination caused rapid reduction in Montgomery-Asberg Depression Rating scale scores, with two of the four studies showing significantly greater improvement than antidepressant monotherapy at study endpoint. Effects of the FLX/OLZ combination were strongest in cases where failure to respond to two antidepressants prior to randomization was established during the current depressive episode. The FLX/OLZ combination was well-tolerated; however, body weight gain and increases in prolactin were greater than that of the antidepressant monotherapy groups, and were comparable to that of OLZ monotherapy. While effective during acute-phase treatment, questions remain regarding the long-term efficacy and safety of FLX/OLZ relative to antidepressant monotherapy and other combination strategies. Efforts aimed at determining the placement of FLX/OLZ among the available options for addressing TRD are limited by lack of comparison and sequential treatment studies. Important aspects of study design and directions for future research are discussed.
\end{abstract}

Keywords: olanzapine, fluoxetine, combination therapy, major depression, treatment resistance

\section{Introduction}

In the last two decades, there has been a marked increase in the number of antidepressant medications available for the treatment of major depressive disorder (MDD). In spite of this, it has become increasingly evident that the effectiveness of antidepressant monotherapy for MDD is much more modest than what was once believed. For example, a recent meta-analysis of all published double-blind placebo controlled antidepressant trials reported an antidepressant response rate of 53\%. ${ }^{1}$ Although this rate of response was significantly greater than the placebo response rate $(36 \%, P<0.05)$, these results have challenged the widely accepted anticipated response rates of $60 \%$ to $70 \%$ reported in prior reviews of controlled studies. ${ }^{2}$ 1500 25th Ave South, Suite 2200, Village at Vanderbilt, Nashville, TN 37212, USA Tel +l 6153277049

Fax +I 6153277093

Email william.v.bobo@vanderbilt.edu 
Rates of acute and sustained symptom remission on antidepressant monotherapy are even lower, both under ideal treatment conditions that characterize randomized controlled trials, and in actual clinical treatment settings. ${ }^{3-5}$ For instance, only about $30 \%$ of patients achieved clinical remission in the first phase of the landmark Sequenced Treatment Alternatives to Relieve Depression (STAR*D) study after 12 weeks of citalopram monotherapy. ${ }^{6}$ Only about $70 \%$ of patients achieved remission after multiple rounds of medications or cognitive therapy, and the majority of patients relapsed within the first year following remission. ${ }^{7}$ The importance of this point rests in the fact that the persistence of residual depressive symptoms is associated with substantial disability and increased risk of a full depressive relapse, even among patients who achieve a positive antidepressant response and remain in active treatment. ${ }^{8,9}$ As such, the majority of antidepressant-treated patients with major depression either fail to improve meaningfully or experience positive but only partial improvement. Clearly, there is an urgent need to develop safe and more effective treatments for major depressive disorder (MDD).

Until such treatments become available, numerous strategies may be applied by the practicing clinician to overcome limitations in the effectiveness of antidepressant monotherapy. In broad terms, these include combining drug treatment with evidence-supported psychotherapies, ${ }^{10}$ combining antidepressants with different pharmacological profiles (combination pharmacotherapy), and combining antidepressants with psychotropic medications that are not antidepressants (augmentation treatment). ${ }^{11,12}$ One such augmentation treatment approach is the combination of antidepressants with atypical antipsychotic drugs. There is now controlled evidence supporting the short-term effectiveness of atypical antipsychotic augmentation of antidepressants, including cases of difficult to treat MDD with and without psychotic features. ${ }^{13-15}$ In addition, one atypical antipsychotic drug, aripiprazole, has a US Food and Drug Administration (FDA) indication as an adjunctive therapy for suboptimal antidepressant response in patients with MDD, ${ }^{16}$ and one selective serotonin reuptake inhibitor (SSRI)/atypical antipsychotic combination (fluoxetine + olanzapine) is FDA approved for acute bipolar depression. ${ }^{17}$ Enthusiasm for this approach is tempered by a lack of long-term effectiveness data, as well as long-term metabolic safety and tolerability concerns for many of these agents. This review is focused on the therapeutic rationale for and clinical evidence supporting the combination of fluoxetine and olanzapine for treatmentresistant MDD (TRD). Characteristics and implications of key design issues in each of the reviewed studies will be emphasized.

\section{Mechanisms of action Neurotransmitter dysfunction in major depression}

A substantial body of evidence indicates that dysfunction in serotonin (5-hydroxytryptamine, 5-HT), norepinephrine (NE) and, to a lesser degree, dopamine (DA) neurotransmission are involved in the pathophysiology of major depression, ${ }^{18-20}$ and that intact 5-HT and NE neurotransmitter systems are needed in order to maintain positive clinical response to antidepressant medications. By now, it is also apparent that early theories that sought to explain the signs and symptoms of major depression solely on the basis of 5-HT and NE depletion have not provided a unifying neurobiological theory explaining why major depression occurs or how antidepressants exert their therapeutic effects. Nevertheless, the monoaminergic neurotransmitter systems have served as the most important pharmacological targets from antidepressant drug development over the last four decades. ${ }^{21}$ Nearly all currently available antidepressant drugs act as potentiators of monoamine neurotransmission, either by inhibition of enzymes needed for monoamine degradation, or by blocking monoamine reuptake sites.

In recent years, a more sophisticated understanding of the pathophysiology of major depression has emerged that focuses on dysregulation of 5-HT and NE transmission rather than monoamine depletion, per se. Abnormalities in the functional activity of numerous brain regions have been identified in depressed patients using functional neuroimaging, including cortical and limbic structures that are critical for regulation of mood, emotional processing, cognitive and psychomotor functioning, and motivation, ${ }^{22-24}$ all common symptoms of depression. Importantly, each of these regions receive serotonergic, noradrenergic and/or dopaminergic projections, ${ }^{25}$ and abnormalities in several of these regions identified on neuroimaging have been shown to resolve following treatment with antidepressants that potentiate 5-HT and/or NE neurotransmission. ${ }^{26-29}$

\section{Limitations of fluoxetine monotherapy}

Monoamine neurotransmission in these key brain regions is tightly regulated, in part, by functional activity of several critical postsynaptic neuroreceptors that are not acted upon directly by SSRIs and other first-line antidepressants. 
Fluoxetine (FLX) and other SSRIs occupy 5-HT reuptake pumps with relatively high affinity and specificity; however, their pharmacological activity is limited, for all practical purposes, to reuptake pump blockade..$^{30,31}$ This poses three potential limitations that may result in a lack of meaningful clinical response to monotherapy with FLX for some patients. First, FLX does not act as a pharmacological agonist and cannot directly activate postsynaptic receptors that are important for the control of monoamine functioning in the central nervous system (CNS). Instead, FLX is dependent almost entirely on intact presynaptic serotonergic functioning. ${ }^{32}$ Second, FLX has only negligible effects on NE and DA reuptake, and does not directly enhance noradrenergic or dopaminergic neurotransmission. ${ }^{30}$ Furthermore, continuous SSRI treatment is associated with suppression of locus coeruleus firing, ${ }^{33-35}$ resulting in decreased NE transmission. This is believed to occur as a consequence of enhanced activation of excitatory postsynaptic 5-HT2A receptors located on inhibitory GABAergic interneurons that synapse with NE neurons. ${ }^{36}$ Third, SSRIs have been shown to suppress ventral tegmental area activity, and therefore reduce DA neurotransmission, ${ }^{37,38}$ possibly by a 5 -HT2C dependent mechanism. ${ }^{39-41}$ Thus, FLX and other SSRIs may not be able to fully optimize central monoaminergic functioning as stand-alone therapies. These limitations cannot be overcome by increases in medication dosage alone.

\section{Augmentation with atypical antipsychotics: focus on olanzapine}

The atypical antipsychotics are a pharmacologically heterogenous group of drugs. Although each is associated with a unique profile of neuroreceptor binding activity, all share the properties of high-potency postsynaptic 5-HT2A antagonism with relatively lower potency dopamine D2 receptor antagonism (or D2 partial agonist effects, in the case of aripiprazole). Importantly, the atypical antipsychotic drug, olanzapine (OLZ), is pharmacologically active at other neuroreceptors that are important for optimizing central monoamine functioning (Table 1). As such, adjunctive therapy with OLZ may be a practical means of overcoming many of the mechanistic limitations of FLX monotherapy, and improving clinical response to FLX in patients with treatment resistant major depression.

For example, 5-HT2A blockade results in numerous effects that are relevant to the activity of FLX. In the presence of 5-HT reuptake inhibition, 5-HT2A receptor antagonism has been shown to enhance 5-HT and NE release in rodents. ${ }^{42}$ In preclinical models, chronic administration of FLX alone

Table I Selected serotonergic neuroreceptor targets of olanzapine with potential relevance for antidepressant augmentation in treatmentresistant depression ${ }^{13,42,45-47}$

\begin{tabular}{|c|c|c|c|}
\hline Neuroreceptor & Pharmacological activity & Functional consequence & Possible benefit in TRD \\
\hline \multirow[t]{3}{*}{ 5-HTIA } & Agonist & Enhance NE release in PFC and NAcc & Improved cognitive functioning \\
\hline & & $\begin{array}{l}\text { Increase DA release in frontal cortex } \\
\text { and NAcc }\end{array}$ & $\begin{array}{l}\text { Improved mood, cognitive functioning and } \\
\text { motivational drive }\end{array}$ \\
\hline & & & $\begin{array}{l}\text { Overcome SSRI induced suppression of central } \\
\text { DA activity }\end{array}$ \\
\hline \multirow[t]{3}{*}{ 5-HT2A } & Antagonist & Enhance $5-\mathrm{HT}$ and NE release ${ }^{\mathrm{a}}$ & $\begin{array}{l}\text { Reverse SSRI induced suppression of locus } \\
\text { coeruleus firing }\end{array}$ \\
\hline & & Enhance DA release in $\mathrm{PFC}^{\mathrm{b}}$ & $\begin{array}{l}\text { Improved regulation of mood and cognitive } \\
\text { functioning }\end{array}$ \\
\hline & & & $\begin{array}{l}\text { Overcome SSRI induced suppression of central } \\
\text { DA activity }\end{array}$ \\
\hline \multirow[t]{3}{*}{$5-\mathrm{HT} 2 \mathrm{C}$} & Antagonist & Enhance NE release in PFC and NAcc & Improved cognitive functioning \\
\hline & & $\begin{array}{l}\text { Increase DA release in frontal cortex } \\
\text { and NAcc }\end{array}$ & $\begin{array}{l}\text { Improved mood, cognitive functioning and } \\
\text { motivational drive }\end{array}$ \\
\hline & & & $\begin{array}{l}\text { Overcome SSRI induced suppression of central } \\
\text { DA activity }\end{array}$ \\
\hline \multirow[t]{2}{*}{$5-\mathrm{HT} 6$} & Antagonist & Enhance DA release in PFC & Improved cognitive functioning \\
\hline & & & $\begin{array}{l}\text { Overcome SSRI induced suppression of central } \\
\text { DA activity }\end{array}$ \\
\hline
\end{tabular}

aln presence of 5-HT reuptake inhibition.

bln presence of weak D2 receptor antagonism.

Abbreviations: TRD, treatment-resistant depression; DA, dopamine; NE, norepinephrine; PFC, ; SSRI, selective serotonin reuptake inhibitor; NAcc, ; 
suppressed locus coeruleus neuronal activity while acutely administered OLZ significantly increased locus coeruleus firing. ${ }^{43}$ The combination of FLX + OLZ, however, resulted in enhanced locus coeruleus firing during both acute and chronic administration. ${ }^{43}$ Increased NE release may be predicted by antagonism of the aforementioned 5-HT2A dependent reduction in NE neuronal firing, while increased NE release may then enhance 5-HT neuronal firing via activation of postsynaptic $\alpha-1$ adrenergic receptors in the raphe nuclei. ${ }^{42}$ In addition, OLZ-associated postsynaptic 5-HT2C antagonism and 5-HT1A activation have also been shown to increase NE release in the prefrontal cortex. ${ }^{44}$ Thus, the combination of FLX + OLZ may result in a greater degree of serotonergic activity than would be expected with FLX alone, and may also reverse FLX-associated NE suppression.

Interactions at serotonin receptors also have important effects on DA neurotransmission. Blockade of 5-HT2A and 5-HT6 receptors, combined with weak D2 receptor antagonism, have both been shown to enhance DA release to a greater degree than blockade of either receptor alone. ${ }^{45,46}$ In addition to increasing NE efflux, OLZ effects on 5-HT2C and 5-HT1A receptors have been shown to increase DA release in the frontal cortex and nucleus accumbens, ${ }^{44,47}$ brain regions that are tied to cognitive functioning and motivational drive. ${ }^{48}$ Clinically, the 5-HT2A antagonists, trazodone and nefazodone, and the 5-HT1A partial agonist, gepirone, have all shown antidepressant effects in clinical trials. ${ }^{49-51}$ Augmentation of FLX with OLZ may therefore provide a means of enhancing DA activity in brain regions implicated in the pathophysiology of major depression that would not be possible with FLX alone, an effect that may be strong enough to overcome FLX-associated suppression of central DA activity and improve antidepressant response.

Finally, there has been considerable interest in the role of brain derived neurotrophic factor (BDNF), one of a number of neurotrophic factors involved in neuronal maintenance and survival, ${ }^{52}$ in the both pathogenesis of major depression and the clinical response to antidepressants. ${ }^{53}$ BDNF has been shown to play an important role in the susceptibility of depression-prone patients to the negative effects of stress. ${ }^{54}$ In post-mortem brains of depressed patients, low levels of hippocampal and prefrontal BDNF have been documented. ${ }^{53}$ Abnormally low levels of serum BDNF have also been found in patients with major depression. ${ }^{55}$ It is noteworthy that long-term exposure to FLX and other antidepressants has been shown to increase BDNF production, ${ }^{56}$ and that stressinduced reduction in levels of BDNF and Bcl-2, another neurotrophic factor, may be blocked with long-term treatment with OLZ. ${ }^{57}$ These results suggest that the combination of FLX + OLZ may have synergistic effects on neurotrophin activity, thereby providing an alternative mechanism by which OLZ may enhance the clinical antidepressive effects of FLX.

\section{Efficacy in clinical trials \\ Results of acute phase studies in TRD}

Five randomized, controlled trials have investigated the effect of OLZ augmentation of FLX in acute phase TRD, two of which were publised in a single paper by Thase et al. ${ }^{64}$ All studies were generally similar in design (Figure 1). Patients with non-psychotic major depression and a prior history of antidepressant failure (variously defined, as discussed below) received an open-label trial of antidepressant monotherapy during a pre-randomization (lead-in) phase. Individuals who failed to achieve a pre-specified threshold of symptom response during this open-label treatment phase were then eligible for randomization to double-blind acute phase treatment with OLZ + FLX or one of several active control conditions, including continuation treatment with the leadin phase antidepressant. The design features and key results of the pre-randomization and randomized acute treatment phases of each study are summarized in Tables 2 and 3, respectively.

The first randomized, controlled study of atypical antipsychotic drug augmentation of antidepressants for TRD compared the effects of FLX + OLZ, FLX monotherapy $(\mathrm{FLX}+$ placebo $)$ and OLZ monotherapy $(\mathrm{OLZ}+$ placebo $) .{ }^{58}$ There is a paucity of long-term efficacy data for the use of FLX/OLZ combination therapy for TRD. In one single-arm study, ${ }^{67}$ a mixed sample of 560 patients with TRD $(\mathrm{N}=145$, defined as having history of past failure to respond to at least two trials of antidepressant treatment using agents of different pharmacological classes) and non-treatment refractory major depression (non-TRD, $\mathrm{N}=407$ ) received an open-label trial. Treatment resistance was confirmed by failure to achieve a partial response to open-label FLX (up to $60 \mathrm{mg} /$ day) during the pre-randomization lead-in phase. Twenty-eight non-responders were then randomly assigned in a double-blind fashion to one of the three acute phase treatment conditions (see Table 3 for mean doses). Continuation of FLX monotherapy yielded no further improvement in depressive symptoms as measured by the Montgomery-Asberg Depression Rating Scale (MADRS), ${ }^{59}$ the Hamilton Depression Rating Scale (HAM-D) ${ }^{60}$ and the Clinical Global Impressions-Depression subscale (CGI-D), ${ }^{61}$ 


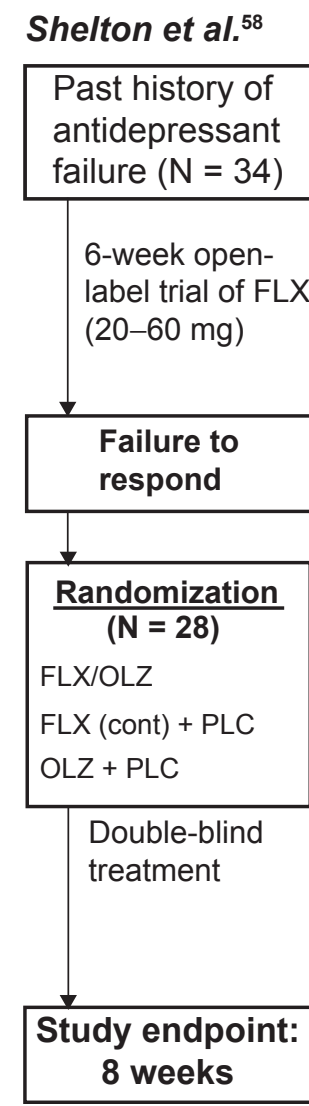

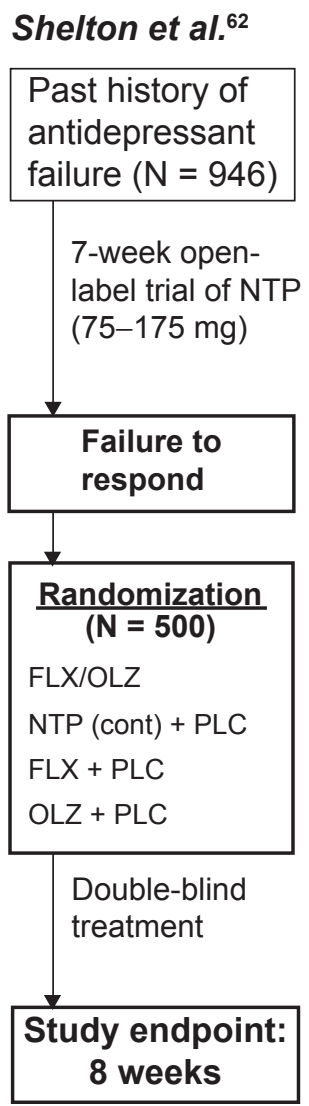

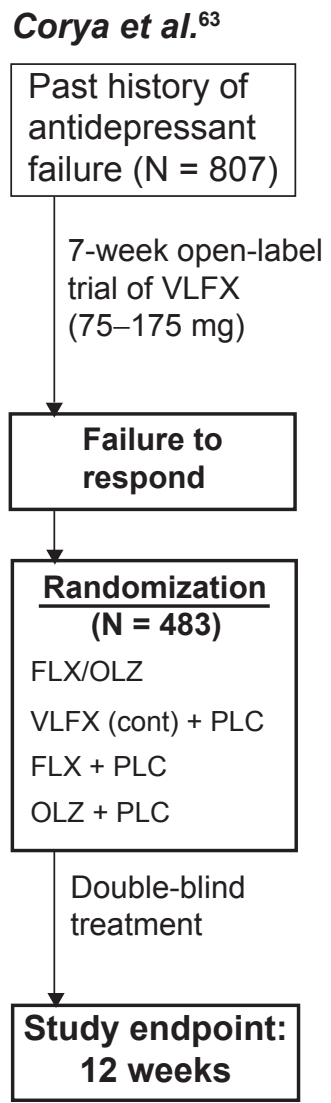

Thase et al. ${ }^{64}$

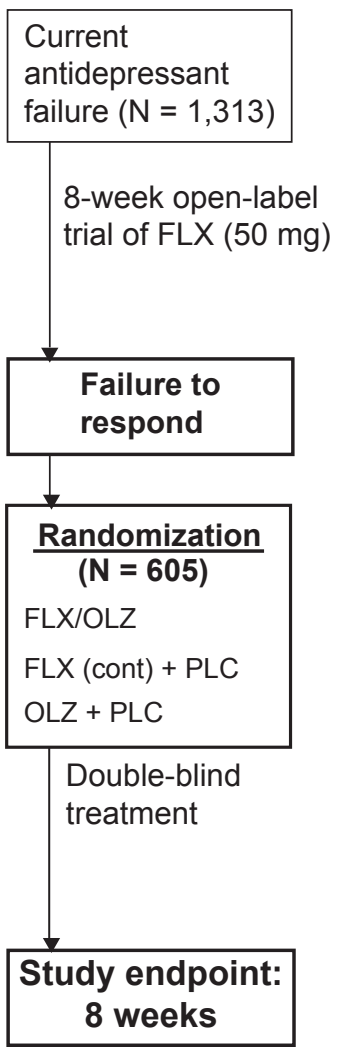

Figure I Basic design features of acute phase studies of combined fluoxetine and olanzapine (FLX/OLZ) in treatment resistant depression. ${ }^{58,62-64}$ Abbreviations: FLX, fluoxetine; FLX (cont), FLX treatment continued into double-blind study phase; FLX/OLZ, fluoxetine + olanzapine combination therapy; NTP, nortriptyline; NTP (cont), NTP treatment continued into double-blind study phase; PLC, placebo;VLFX, venlafaxine.

while OLZ monotherapy achieved a modest benefit over FLX alone. The FLX/OLZ combination resulted in significantly greater improvement in depressive symptoms than FLX or OLZ alone (Table 3). The proportion of patients who achieved positive clinical response ( $\geq 50 \%$ improvement in MADRS scores at endpoint) was greater for the FLX/OLZ group (60\%) compared with OLZ (0\%) and FLX (10\%) monotherapy groups. Pairwise comparisons of the proportion of those achieving positive clinical response were significant only for FLX/OLZ vs OLZ monotherapy, favoring combination therapy.

Four larger-scale randomized, double-blind multi-center studies investigating the effects of FLX/OLZ combination therapy were then completed. ${ }^{62-64}$ In the first study, ${ }^{62}$ 500 non-psychotically depressed patients with a prior history of failure to respond to $\mathrm{a} \geq 4$ week trial of SSRI treatment and prospective failure of open-label NTP (Table 2) were randomized to one of four treatment groups for the 8-week acute phase study: FLX + OLZ, FLX monotherapy (FLX + placebo), OLZ monotherapy (OLZ + placebo), or continuation treatment with NTP (+ placebo) at the same dosage used during the pre-randomization lead-in phase (Table 3). The OLZ/FLX combination produced rapid antidepressant effect and statistically superior improvement in MADRS scores compared with all monotherapy groups through the first four weeks of treatment; however, there were no significant differences between these groups at study endpoint. There were also no significant differences between the FLX + OLZ, FLX monotherapy, OLZ monotherapy, or NTP monotherapy groups in Hamilton Anxiety Scale (HAM-A) ${ }^{65}$ or Clinical Global ImpressionsIllness Severity subscale scores, ${ }^{61}$ or rates of categorical treatment response $(27.5 \%, 28.9 \%, 19.3 \%$, and $30.3 \%$, respectively) or remission $(16.9 \%, 13.3 \%, 12.9 \%$, and $18.2 \%$, respectively), at study endpoint.

The second large-scale study compared the clinical effects of OLZ/FLX combination, ${ }^{63}$ FLX monotherapy, OLZ monotherapy, and VLFX monotherapy over 12 weeks in a cohort of non-psychotically depressed patients $(\mathrm{N}=483)$ with a past history of failure to respond to a $\geq 6$ week therapeutic SSRI trial and prospective failure of an openlabel lead-in trial of VLFX (Table 2). Again, there was 


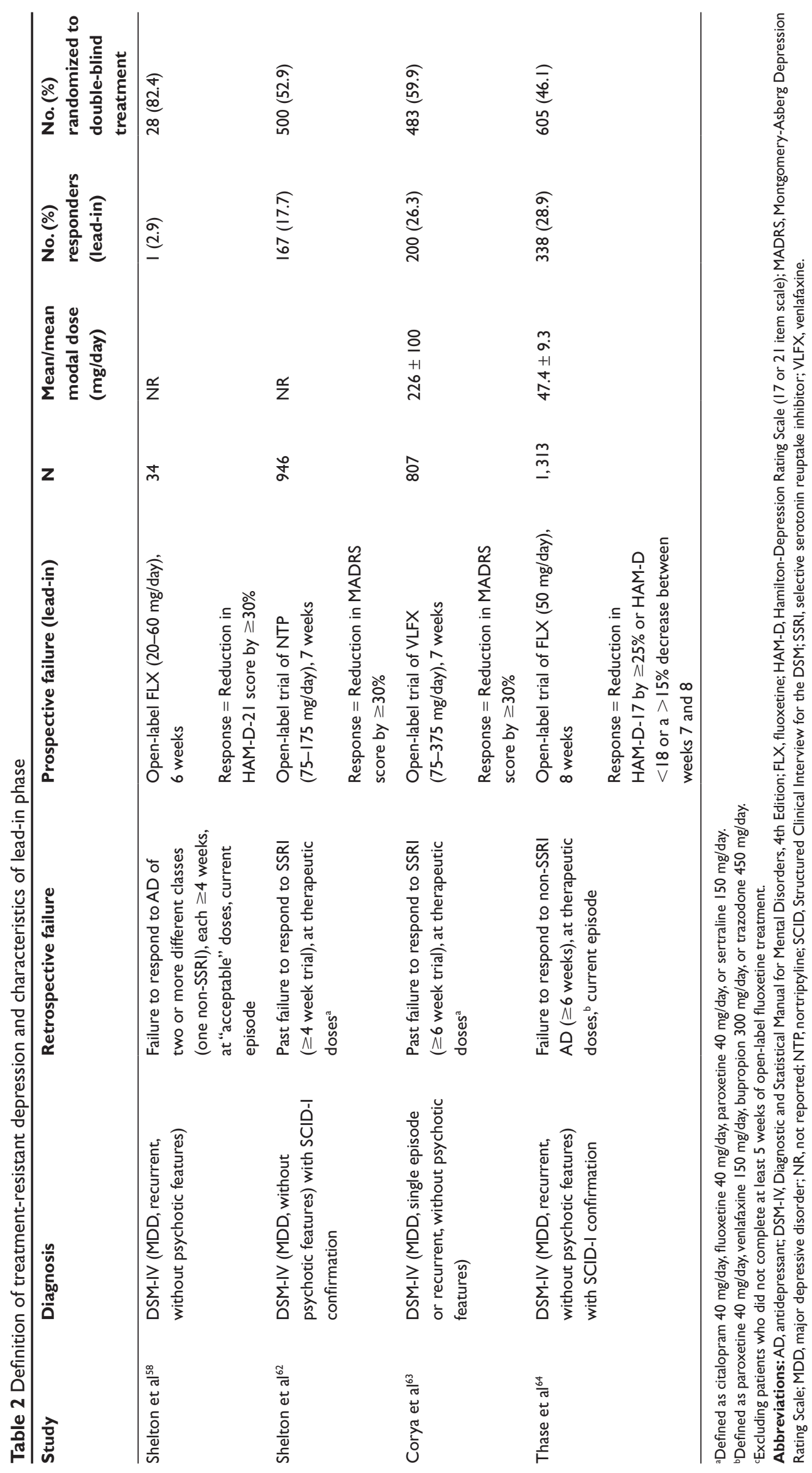




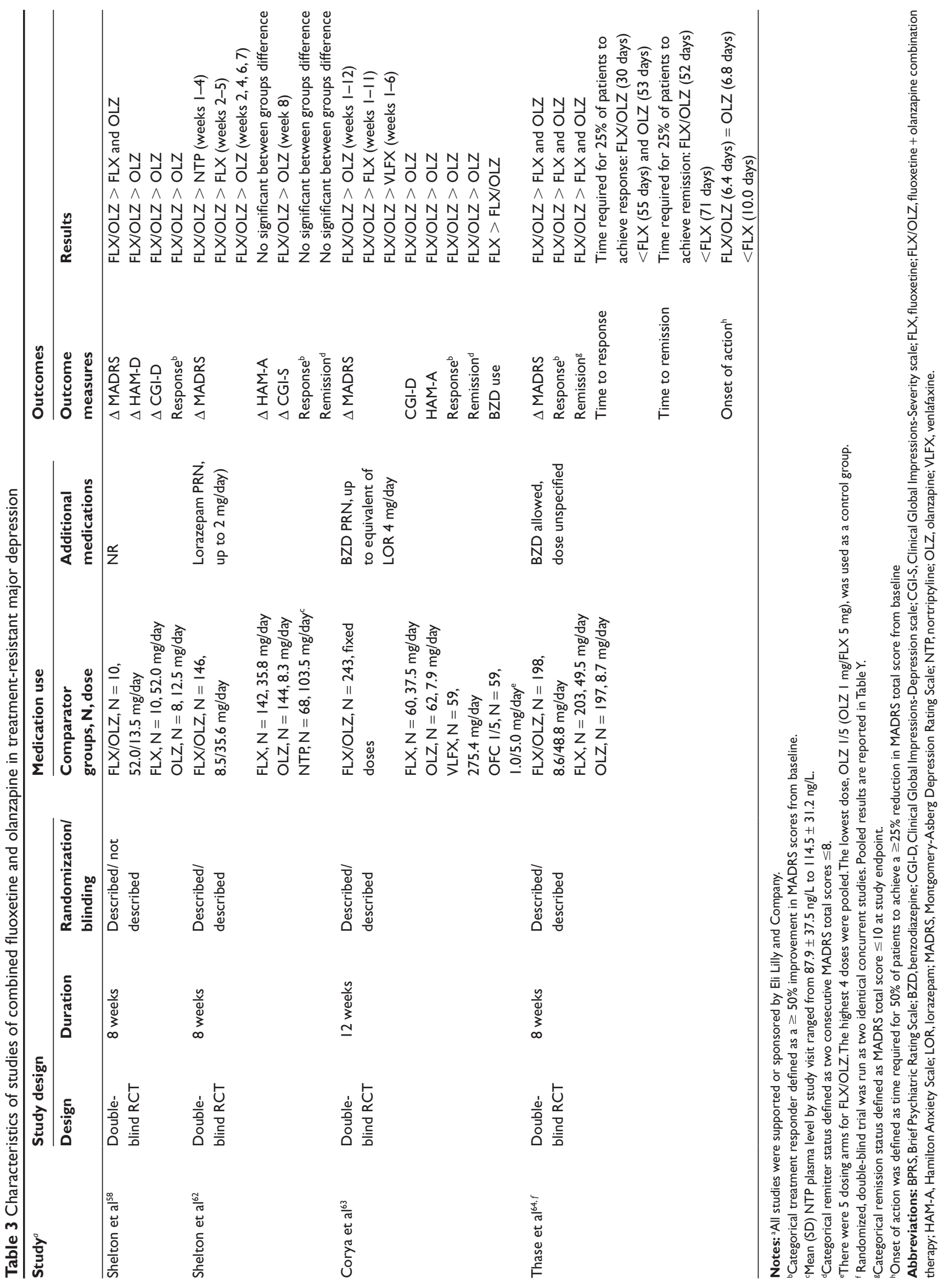


significant early improvement associated with OLZ/FLX treatment, and significantly greater improvement in MADRS scores for OLZ/FLX over VLFX monotherapy (first 6 weeks only) and FLX monotherapy (first 11 weeks only). At study endpoint, there was no evidence of a FLX/OLZ advantage over any antidepressant monotherapy group (Table 3). This included no significant differences between FLX + OLZ, FLX monotherapy, OLZ monotherapy, and VLFX monotherapy groups in rates of categorical treatment response $(43.3 \%, 33.9 \%, 25.4 \%$, and $50.0 \%$, respectively) or remission $(29.9 \%, 17.9 \%, 13.6 \%$, and $22.4 \%$, respectively) at study endpoint.

The final two studies were of identical design and were run concurrently (Study 1 and Study 2). Results were reported separately and as pooled data. ${ }^{64}$ Both studies were randomized, double-blind 8-week comparisons of FLX/OLZ combination therapy, FLX monotherapy $($ FLX + placebo $)$ and OLZ monotherapy (OLZ + placebo $)$ in a cohort of patients with non-psychotic major depression ( $\mathrm{N}=605$, pooled data set). Eligibility was based on a past history of SSRI failure during the current depressive episode only, followed by prospective failure of an 8-week open-label FLX (50 mg/day) lead-in trial. In Study 1, there was no evidence of a superior antidepressive effect for FLX/OLZ combination treatment over either monotherapy group. However, in Study 2, the FLX/OLZ combination resulted in significantly greater improvement in MADRS scores, and in categorical response and remission rates, compared with both monotherapy groups at study endpoint. The pooled analysis of both projects revealed significantly greater improvement in MADRS scores, higher rates of categorical response (FLX + OLZ, 40.4\%; FLX, 29.6\%; OLZ, 25.9\%) and remission (FLX + OLZ, 27.3\%; FLX $16.7 \%$; OLZ 14.7\%), and shorter time required for $25 \%$ of patients to achieve therapeutic responder status (FLX + OLZ, 30 days; FLX, 55 days; OLZ, 53 days), compared with both monotherapy groups (Table 3 ). There were also advantages for FLX/OLZ over both antidepressant monotherapies in family and leisure functioning as measured by the Sheehan Disability Scale. ${ }^{66}$ There was no statistically significant advantage observed for work functioning.

\section{Results of long-term studies}

There is a paucity of long-term efficacy data for the use of FLX/OLZ combination therapy for TRD. In one single-arm study, ${ }^{67}$ a mixed sample of 560 patients with TRD $(\mathrm{N}=145$, defined as having history of past failure to respond to at least two trials of antidepressant treatment using agents of different pharmacological classes) and non-treatment refractory major depression (non-TRD, $\mathrm{N}=407$ ) received an open-label trial FLX/OLZ (mean modal dose $=46.1 \pm 20.7 \mathrm{mg}$ /day of FLX/7.5 $\pm 3.5 \mathrm{mg} /$ day of OLZ) for up to 76 weeks. There was a significant reduction in MADRS and CGI-S subscale scores as early as 0.5 weeks, followed by strong and continuous reductions in these measures througout the remainder of the study in both TRD and non-TRD patients. At study endpoint, $61.6 \%$ of patients met categorical response criteria ( $\geq 50 \%$ reduction in MADRS scores), and $56.3 \%$ of patients achieved remission (MADRS scores of $\leq 8$ on two consecutive visits. Higher remission rates (60.7\% vs $44.1 \%)$ and shorter time to remission were observed in the non-TRD compared with TRD sample. These results were tempered by relatively high droput rates. Only 177 (31.6\%) of patients completed 52 weeks of treatment, and only $143(25.5 \%)$ of patients completed the 76-week trial. In addition, of the patients who achieved remission, $12.1 \%$ of non-TRD and $25.0 \%$ of TRD patients relapsed (defined as a MADRS score of $\geq 16$ at any two visits following remission). More longterm studies are needed.

\section{Tolerability and safety}

Safety and tolerability results across each of the acute-phase studies are summarized in Table 4. Rates of discontinuation due to treatment-emergent adverse effects (TEAEs) were greatest for FLX/OLZ- and OLZ monotherapy-treated patients. Because those who did not tolerate lead-in antidepressant medication were dropped prior to randomization, discontinuation rates tended to be lowest for drugs that were utilized during the lead-in phase and were continued during the double-blind treatment period.

Increases in body weight, total non-fasting cholesterol and serum prolactin (PRL) levels were greatest for the FLX/OLZ and OLZ groups. There was little change from baseline in any of these measures for the antidepressant monotherapy groups during double-blind treatment. The magnitude of increases in these measures between FLX/ OLZ and OLZ groups was generally similar. However, increases in total cholesterol were significantly greater for FLX + OLZ vs OLZ monotherapy in a pooled analysis of data from the 5 acute phase studies reviewed above. ${ }^{76}$ There were no significant differences for change in triglyceride levels between these two groups in the Thase et al ${ }^{64}$ study. There were no significant between groups differences in baseline to endpoint change in non-fasting glucose in the studies by Shelton et $\mathrm{al}^{62}$ and Thase et al; ${ }^{64}$ however, in both studies, the numerical increases in non-fasting glucose 


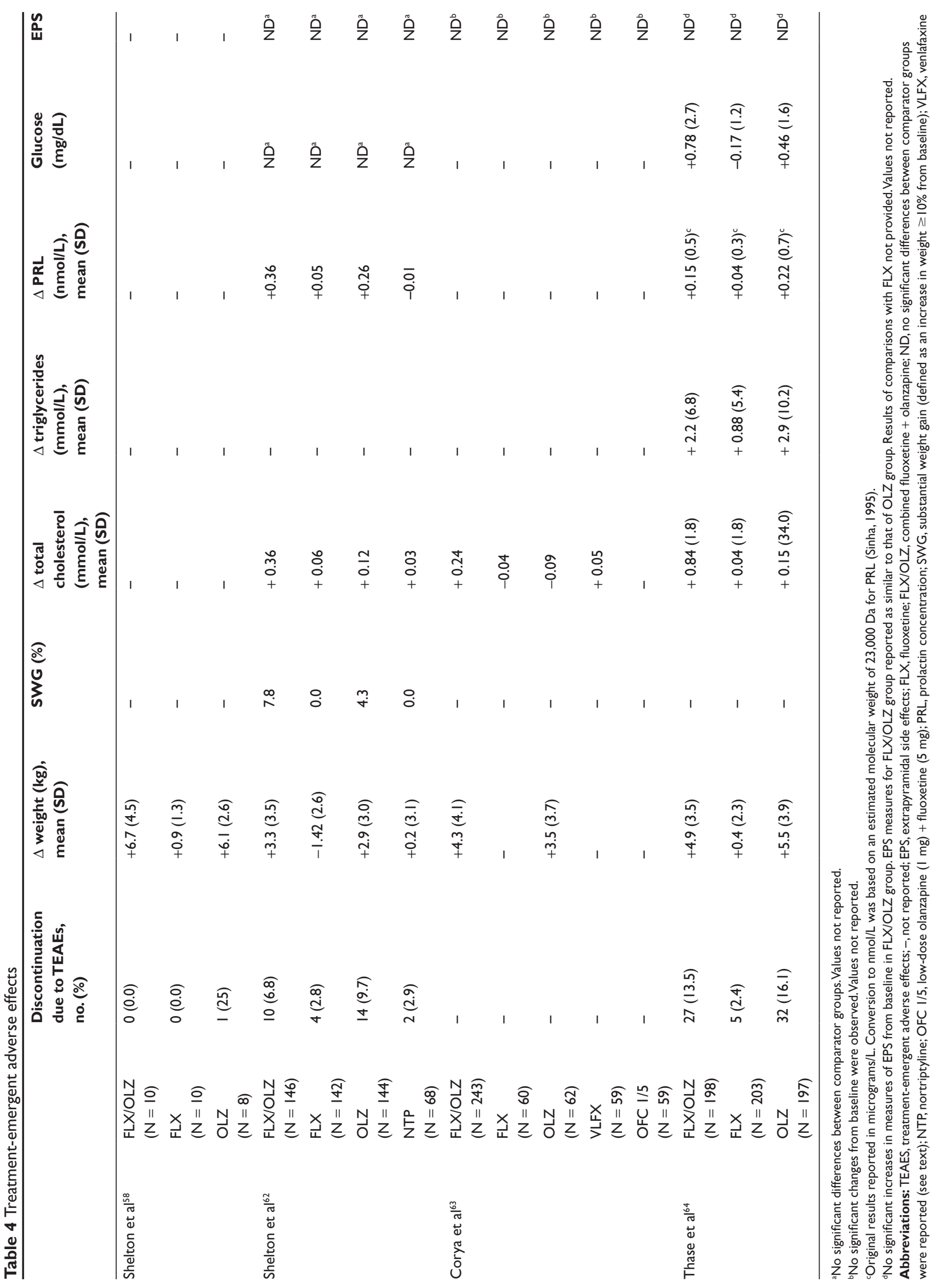


levels were greatest in the FLX/OLZ and OLZ groups relative to the antidepressant monotherapy groups. Similar increases in weight $(+5.6 \pm 6.6 \mathrm{~kg})$ and non-fasting glucose levels were reported in the long-term open-label study by Corya et $\mathrm{al}^{67}$

Extrapyramidal side effects (EPS) were measured using the Simpson-Angus Scale (SAS), ${ }^{68}$ Barnes Akathisia Scale (BARS), ${ }^{69}$ and the Abnormal Involuntary Movement Scale $(\mathrm{AIMS})^{70}$ in each of the reviewed studies. There were no significant between groups differences in EPS measures in the Shelton et al ${ }^{62}$ study. There were no significant changes in these measures from baseline in any of the treatment groups in the Corya et $\mathrm{l}^{63}$ study, or in the FLX/OLZ group in the Thase et $\mathrm{al}^{64}$ study. EPS measures for the FLX/OLZ group were reported as being similar to that of the OLZ group. In the long-term open-label study by Corya et $\mathrm{al}^{67}$ there were no significant increases in SAS, BARS or AIMS scores between baseline and the 76-week study endpoint. In the only study that assessed PRL response, FLX/OLZ was associated with significantly greater increases in PRL concentration than FLX, OLZ or NTP monotherapy. ${ }^{62}$

\section{Discussion}

\section{Clinical issues}

TRD is a common occurrence in clinical practice, and poses numerous challenges for the practicing clinician. While atypical antipsychotic augmentation of antidepressant medication was shown to be an effective strategy for patients with TRD in a recent meta-analysis of 10 randomized, doubleblind, placebo controlled trials, ${ }^{14}$ their exact place within the mélange of therapeutic options for managing TRD has not been precisely defined. For this to be determined, data from sequential treatment studies and/or head-to-head comparisons would be needed. The largest sequential investigation of therapeutic options in TRD to date, the Sequenced Treatment Alternatives to Relieve Depression (STAR*D) study, did not investigate the effects of atypical antipsychotic augmentation. Data from head-to-head comparison trials between FLX/OLZ and other interventions for TRD are not yet available. Nevertheless, based on clinical trial results that are currently available, the strongest clinical evidence supporting a role for atypical antipsychotic drugs as augmenters of antidepressants in TRD exists for olanazapine, quetiapine and aripiprazole, at least for short-term, acute phase treatment. ${ }^{13,14,71-74}$ Of these, only aripiprazole is currently approved for this indication.

Our review of the literature, which focused on the effects of OLZ augmentation of FLX in TRD, also supports the short-term efficacy of this strategy, is consistent with a prior narrative review of FLZ $+\mathrm{OLZ}$ in TRD. ${ }^{75}$ Effectiveness of this combination appears to be particularly strong for those with an inadequate SSRI response and treatment resistance in the current depressive episode. Focusing on this subpopulation of patients has at least two advantages. First, the decision to augment antidepressant therapy with OLZ in actual clinical practice would be based on poor treatment response to antidepressant monotherapy in the current depressive episode, rather than having a history of poor antidepressant response in prior depressive episodes. Next, the consideration of only those subjects who met treatment failure criteria during the current depressive episode would guard against the inclusion of less treatment resistant cases in studies that focus specifically on intervention effects in TRD. This would result in a sample that is more representative of the TRD population encountered in clinical practice. Patients with TRD, defined in this more stringent manner, would also have a greater likelihood of achieving a more favorable clinical response to antidepressant augmentation compared with monotherapy. This position is supported by results of Shelton et al, ${ }^{58}$ Thase et $\mathrm{al}^{64}$ and the pooled analysis by Trivedi et $\mathrm{al}^{76}$ which focused on patients with TRD based on the current depressive episode only. All showed stronger effects of FLX/OLZ augmentation relative to antidepressant monotherapy than did individual large-scale studies that used a less stringent definition of TRD. ${ }^{62,63}$

Available evidence of effectiveness FLX + OLZ in TRD is similar to the published data describing the combination's effectiveness for the short-term acute-phase treatment of depression in patients with bipolar I disorder. ${ }^{77,78}$ In two randomized, controlled studies, FLX + OLZ resulted in greater 8-week improvement in depressive symptomatology compared with placebo and OLZ monotherapy, ${ }^{79}$ and greater 7-week improvement than lamotrigine. ${ }^{78}$ In addition, combination therapy with FLX + OLZ was associated with higher remission rates than placebo or OLZ monotherapy, ${ }^{77}$ and a similar remission rate as lamotrigine. ${ }^{78}$ In one small placebocontrolled study comparing the effects of FLX + OLZ, OLZ monotherapy, FLX monotherapy and placebo in depressed patients with bipolar I or II disorder, there were significant reductions in depressive symptoms for the entire cohort; however, the study was under-powered to detect differences between treatment groups. ${ }^{80}$ In published studies of bipolar depressed patients, FLX + OLZ was generally well tolerated, with limited safety data suggesting that FLX + OLZ combination therapy was associated with weight gain and lipid and prolactin level elevation similar to that of OLZ monotherapy. As is the case with TRD, long-term safety and effectiveness 
data in bipolar patients are generally lacking. FLX + OLZ combination therapy was associated with significantly greater improvement in depressive symptoms than lamotrigine in a 25-week double blind extension of the 7-week acute phase study by Brown et al. ${ }^{78,79}$ However, FLZ + OLZ was associated with more frequent weight gain and significantly higher incidence of clinically significant hypercholesterolemia ( $\geq 240 \mathrm{mg} / \mathrm{dL}$ ) and weight gain ( $\geq 7 \%$ increase in weight from baseline) at the 25 week endpoint. ${ }^{79}$ Overall, the state of the available evidence for FLX + OLZ therapy in bipolar depression is similar to that of the combination in TRD, with encouraging results shown in short-term acute phase studies, but limited longer-term data.

Enthusiasm is thus tempered by several unanswered questions regarding the use of FLX/OLZ augmentation in TRD. The optimal augmenting dose of OLZ has not been established, though anecdotal reports and uncontrolled evidence suggest that the target dose may be lower (2.5 to $10 \mathrm{mg}$ ) than those used in the treatment of schizophrenia or bipolar disorder. ${ }^{81,82}$ The long-term efficacy and safety profile of FLX/OLZ in TRD has also not been established. There have been no controlled investigations longer than 12 weeks to date. As such, there are also no studies focused on the question of how long OLZ augmentation of FLX is required among patients who respond acutely to the combination.

The paucity of long-term studies is also of vital clinical interest because of concerns about body weight gain and dysmetabolic effects that are associated with OLZ. Of the antipsychotic drugs, OLZ is among the most orexigenic and, with the exception of clozapine, is associated with the greatest short-term weight gain and longer-term risk of hyperglycemia, diabetes, and atherogenic dyslipidemia. ${ }^{83-85}$ These metabolic risks must be carefully considered when deciding among the varied options available for augmentation of antidepressants in TRD. Apart from the obvious long-term health concerns, individuals with MDD are in physically poorer health than the general population ${ }^{86}$ and therefore comprise a patient sub-population that is already at risk for profound cardiovascular morbidity and early mortality. Indeed, major depression has been identified as an independent risk factor for coronary artery disease and premature death due to cardiovascular causes ${ }^{87-89}$ As is the case with schizophrenia and bipolar disorder, psychiatric illnesses that are associated with considerable physical health burden and early mortality due to cardiovascular disease, ${ }^{90,91}$ use of OLZ for patients with major depression requires close metabolic monitoring and follow-up. The stringency of such monitoring should generally be the same that used for OLZ treatment of bipolar disorder and schizophrenia, even if lower doses of OLZ are shown to be effective for antidepressant augmentation.

\section{Characteristics and implications of study design and execution}

The definition of TRD is a matter of ongoing debate, and numerous and varying criteria have been used to defined TRD samples in clinical trials. ${ }^{92}$ To improve uniformity, several definitional models have been proposed, a detailed review of which will not be provided here. The interested reader is referred to several excellent reviews. ${ }^{93-95}$ For purposes of the current discussion, some design features and issues in study execution, many of which focus on sample selection and study definitions of TRD, may have led to the inclusion of less-treatment resistant depressed patients. This could have introduced bias toward null findings in some of the reviewed studies.

For example, two of the reviewed studies based their definition of TRD, in part, on a history of failure not confined to the current depressive episode. ${ }^{62,63}$ Only the studies by Shelton et $\mathrm{al}^{58}$ and Thase et $\mathrm{al}^{64}$ randomized patients that had both a retrospective and prospective lead-in antidepressant failure in the current depressive episode. Only the Thase et $\mathrm{al}^{64}$ group randomized patients that had both a retrospective and prospective lead-in antidepressant failure in the current depressive episode only. Planned sub-group analyses of subjects in the Shelton et $\mathrm{al}^{62}$ and Corya et $\mathrm{al}^{63}$ studies who met both retrospective and prospective TRD inclusion criteria for the current depressive episode revealed a more marked treatment effect for FLX/OLZ. In the Corya et $\mathrm{al}^{63}$ study, improvement in MADRS at study endpoint was significantly greater for FLX/OLZ compared with FLX alone in this patient subgroup. A pooled analysis of data from all of the above reviewed acute-phase studies was performed by Trivedi et $\mathrm{al}^{76}$ which focused on only those subjects with a history of antidepressant non-response in the current depressive episode $(\mathrm{N}=1146)$. FLX/OLZ was associated with significantly greater 8-week improvement in MADRS scores $(-13.0$ vs -8.6 vs -8.2$)$ and higher rates of remission ( $25.5 \%$ vs $17.3 \%$ vs $14.0 \%$ ) compared with FLX or OLZ monotherapy. ${ }^{76}$

Second, although a 4- to 6-week time interval for leadin phase treatment could be considered generally adequate, many patients may require longer than this to adequately respond to antidepressant monotherapy, ${ }^{96}$ a situation that may be even more problematic for drugs that require slower or more prolonged titration to therapeutic doses. These factors may have been particularly relevant for the Shelton et $\mathrm{al}^{62}$ and Corya et $\mathrm{al}^{63}$ studies, both of which utilized antidepressant medications during the lead-in phase that required titration to 
therapeutic doses (NTP and VLFX, respectively). Assuming that dosage adjustments were made during the first week even after achieving initial target doses, this would have left approximately 6 weeks of lead-in antidepressant treatment at adequate dosage. This time interval would not be sufficient to exclude late responders who, by definition, are not treatment-resistant but would have still been included in the double-blind acute treatment phase of these studies.

Third, investigators in the Shelton et $\mathrm{a}^{62}$ and Corya et $\mathrm{al}^{63}$ studies were not blinded to the double-blind acute phase entry criteria (reduction of $\geq 30 \%$ in MADRS scores) which, as pointed out by the study authors, may have led to unconscious bias toward continued treatment and randomization via an under-rating of MADRS scores at the end of the lead-in phase. The lower than expected rates of partial treatment response at the end of the lead-in phase of both of these studies (18\% and 26\%, respectively) support this hypothesis; however, response rates for individuals with treatment resistant depression would also be expected to be low. In the study by Thase et $\mathrm{al}^{64}$ investigators were blinded to the double-blind acute phase study entry criteria. As such, the potential limitations posed by lack of blinding to acute phase study criteria do not apply to this study.

Fourth, in each of the reviewed studies, some patients who were eligible for double-blind acute phase treatment were re-randomized to the same antidepressant medication that they received during the lead-in period. These individuals would have received up to 15 weeks of NTP treatment in the Shelton et $\mathrm{al}^{62}$ study, up to 19 weeks of VLFX treatment in the Corya et $\mathrm{al}^{63}$ study, and up to 16 weeks of FLX treatment in the Thase et $\mathrm{al}^{64}$ study, thus causing an imbalance in study design and possible bias in favor of NTP, VLFX, or FLX, respectively. Finally, none of the reviewed studies included a placebo, as it was deemed unethical to deny active treatment to patients with refractory depression. Lack of a placebo group, however, makes it difficult to rule out non-pharmacological causes of clinical improvement across groups.

\section{Summary and conclusions}

TRD represents a clinical challenge for which more effective treatments are urgently needed. The combination of FLX and OLZ has shown considerable promise in short-term, acute phase studies; however, little is known about the relative effectiveness of FLX/OLZ augmentation compared with other augmenting or combination strategies for TRD. Several questions remain concerning the optimal dosing and required duration of OLZ in combination with FLX for patients with TRD, and little is known about the long-term efficacy, tolerability and safety of this combination. This includes lack of data regarding long-term effects of FLX/OLZ on surrogate markers of cardiovascular risk (weight gain, dysglycemia, atherogenic dyslipidemia) and important clinical endpoints (eg, development of diabetes and serious cardiovascular disease). Future studies of comparative effectiveness of FLX/OLZ augmentation and other forms of combination therapy for TRD are needed in order to best determine its place among existing treatment options, either in the form of head-to-head comparisons with other TRD treatment options or as a therapeutic option in sequential treatment studies of patients with major depression.

\section{Information sources}

PubMed, PsycINFO, Ovid MEDLINE, clinicaltrials.gov.

\section{Disclosures}

Dr Bobo has received grant/research support from Cephalon and has served on the speaker's bureau in the past for Pfizer and Janssen Pharmaceutica.

Dr Shelton has received grant/research support from Eli Lilly and Company, GlaxoSmithKline, Janssen Pharmaceutica, Pfizer, Inc., Sanofi-Aventis, Wyeth, Inc., AstraZeneca Pharmaceuticals, Forest Pharmaceuticals, Otsuka America, Inc., Pamlab, Inc., and Abbott Laboratories; has served as a paid consultant for Pfizer, Inc., Janssen Pharmaceutica, Eli Lilly and Co., Forest Pharmaceuticals, Otsuka America, Inc., Pamlab Inc., and Sierra Neuropharmaceuticals; and has served on the speaker's bureau in the past for Bristol-Myers Squibb, Eli Lilly and Company, Janssen Pharmaceutica, Pfizer, Inc., GlaxoSmithKline, Wyeth, Inc., and Pamlab, Inc.

\section{References}

1. Papakostas GI, Fava M. Does the probability of receiving placebo influence clinical trial outcome? A meta-regression of double-blind, randomized clinical trials in MDD. Eur Neuropsychopharmacol. 2009;19(1):34-40.

2. Shelton RC. Treatment options for refractory depression. J Clin Psychiatry. 1999;60 Suppl 4:57-61.

3. Petersen T, Papakostas GI, Posternak MA, et al. Empirical testing of two models for staging antidepressant treatment resistance. J Clin Psychopharmacol. 2005 August; 25(4):336-341.

4. Machado M, Iskedjian M, Ruiz I, Einarson TR. Remission, dropouts, and adverse drug reaction rates in major depressive disorder: a meta-analysis of head-to-head trials. Curr Med Res Opin. 2006;22(9):1825-1837.

5. Moller HJ. Outcomes in major depressive disorder: the evolving concept of remission and its implications for treatment. World J Biol Psychiatry. 2008;9(2):102-114.

6. Trivedi MH, Rush AJ, Wisniewski SR, et al. Evaluation of outcomes with citalopram for depression using measurement-based care in STAR*D: implications for clinical practice. Am J Psychiatry. 2006;163(1):28-40.

7. Rush AJ, Trivedi MH, Wisniewski SR, et al. Acute and longer-term outcomes in depressed outpatients requiring one or several treatment steps: a STAR*D report. Am J Psychiatry. 2006;163(11):1905-1917. 
8. Greden JF. The burden of disease for treatment-resistant depression. J Clin Psychiatry. 2001;62 Suppl 16:26-31.

9. Keller MB, Boland RJ. Implications of failing to achieve successful long-term maintenance treatment of recurrent unipolar major depression. Biol Psychiatry. 1998;44(5):348-360.

10. Thase ME, Friedman ES, Biggs MM, et al. Cognitive therapy versus medication in augmentation and switch strategies as second-step treatments: a STAR*D report. Am J Psychiatry. 2007;164(5):739-752.

11. Thase ME, Rush AJ. When at first you don't succeed: sequential strategies for antidepressant nonresponders. J Clin Psychiatry. 1997;58 Suppl 13:23-29.

12. Trivedi MH, Fava M, Wisniewski SR, et al. Medication augmentation after the failure of SSRIs for depression. $N$ Engl J Med. 2006;354(12): 1243-1252.

13. Shelton RC, Papakostas GI. Augmentation of antidepressants with atypical antipsychotics for treatment-resistant major depressive disorder. Acta Psychiatr Scand. 2008;117(4):253-259.

14. Papakostas GI, Shelton RC, Smith J, Fava M. Augmentation of antidepressants with atypical antipsychotic medications for treatmentresistant major depressive disorder: a meta-analysis. J Clin Psychiatry. 2007;68(6):826-831.

15. Tyrka AR, Price LH, Mello MF, Mello AF, Carpenter LL. Psychotic major depression: a benefit-risk assessment of treatment options. Drug Saf. 2006;29(6):491-508.

16. Nelson JC, Pikalov A, Berman RM. Augmentation treatment in major depressive disorder: focus on aripiprazole. Neuropsychiatr Dis Treat. 2008;4(5):937-948.

17. Deeks ED, Keating GM. Olanzapine/fluoxetine: a review of its use in the treatment of acute bipolar depression. Drugs. 2008;68(8): 1115-1137.

18. Jans LA, Riedel WJ, Markus CR, Blokland A. Serotonergic vulnerability and depression: assumptions, experimental evidence and implications. Mol Psychiatry. 2007;12(6):522-543.

19. Schidkraut JJ. The catecholamine hypothesis of affective disorders a review of supporting evidence. Am J Psychiatry. 1965;122:509-522.

20. Elhwuegi AS. Central monoamines and their role in major depression. Prog Neuropsychopharmacol Biol Psychiatry. 2004;28(3):435-451.

21. Richelson E. Interactions of antidepressants with neurotransmitter transporters and receptors and their clinical relevance. J Clin Psychiatry. 2003;64 Suppl 13:5-12.

22. Liotti M, Mayberg HS, McGinnis S, Brannan SL, Jerabek P. Unmasking disease-specific cerebral blood flow abnormalities: mood challenge in patients with remitted unipolar depression. Am J Psychiatry. 2002;159(11):1830-1840.

23. Mayberg HS, Liotti M, Brannan SK, et al. Reciprocal limbic-cortical function and negative mood: converging PET findings in depression and normal sadness. Am J Psychiatry. 1999;156(5):675-682.

24. Drevets WC, Bogers W, Raichle ME. Functional anatomical correlates of antidepressant drug treatment assessed using PET measures of regional glucose metabolism. Eur Neuropsychopharmacol. 2002;12(6):527-44.

25. Marek G, Duman RS. Neural circuitry and signaling in depression. In: Kaplan GB, Hammer RP, editors. Brain Circuitry and Signaling in Psychiatry. Washington, DC: American Psychiatric Publishing, Inc.; 2002. p. 153-178.

26. Walsh ND, Williams SC, Brammer MJ, et al. A longitudinal functional magnetic resonance imaging study of verbal working memory in depression after antidepressant therapy. Biol Psychiatry. 2007 1;62(11):1236-1243.

27. Ishizaki J, Yamamoto H, Takahashi T, Takeda M, Yano M, Mimura M. Changes in regional cerebral blood flow following antidepressant treatment in late-life depression. Int J Geriatr Psychiatry. 2008;23(8): 805-811.

28. Vlassenko A, Sheline YI, Fischer K, Mintun MA. Cerebral perfusion response to successful treatment of depression with different serotoninergic agents. J Neuropsychiatry Clin Neurosci. 2004;16(3): $360-363$
29. Davies J, Lloyd KR, Jones IK, Barnes A, Pilowsky LS. Changes in regional cerebral blood flow with venlafaxine in the treatment of major depression. Am J Psychiatry. 2003;160(2):374-376.

30. Tatsumi M, Groshan K, Blakely RD, Richelson E. Pharmacological profile of antidepressants and related compounds at human monoamine transporters. Eur J Pharmacol. 1997;340(2-3):249-258.

31. Cusack B, Nelson A, Richelson E. Binding of antidepressants to human brain receptors: focus on newer generation compounds. Psychopharmacology (Berl). 1994;114(4):559-565.

32. Rosenbaum JF, Tollefson GD. Fluoxetine. In: Schatzbert AF, NemeroffCB, editors. Textbook of Psychopharmacology. Third Edition ed. Arlington, VA: American Psychiatric Publishing, Inc.; 2004. p. 231-246.

33. Szabo ST, de MC, Blier P. Modulation of noradrenergic neuronal firing by selective serotonin reuptake blockers. Br J Pharmacol. 1999;126(3):568-571.

34. Sodero AP, Valdomero A, Cuadra GR, Ramirez OA, Orsingher OA. Locus coeruleus activity in perinatally protein-deprived rats: effects of fluoxetine administration. Eur J Pharmacol. 2004;503(1-3):35-42.

35. West CH, Ritchie JC, Boss-Williams KA, Weiss JM. Antidepressant drugs with differing pharmacological actions decrease activity of locus coeruleus neurons. Int J Neuropsychopharmacol. 2009;12(5): 627-641.

36. Szabo ST, Blier P. Functional and pharmacological characterization of the modulatory role of serotonin on the firing activity of locus coeruleus norepinephrine neurons. Brain Res. 2001;922(1):9-20.

37. Prisco S, Esposito E. Differential effects of acute and chronic fluoxetine administration on the spontaneous activity of dopaminergic neurones in the ventral tegmental area. Br J Pharmacol. 1995;116(2):1923-1931.

38. DiMascio M, DiGiovanni G, DiMatteo V, Prisco S, Esposito E. Selective serotonin reuptake inhibitors reduce the spontaneous activity of dopaminergic neurons in the ventral tegmental area. Brain Res Bull. 1998;46(6):547-554.

39. DiGiovanni G, DeDeurwaerdere P, DiMascio M, DiMatteo V, Esposito E, Spampinato U. Selective blockade of serotonin-2C/2B receptors enhances mesolimbic and mesostriatal dopaminergic function: a combined in vivo electrophysiological and microdialysis study. Neuroscience. 1999;91(2):587-597.

40. DiMatteo V, DiGiovanni G, DiMascio M, Esposito E. SB 242084, a selective serotonin2C receptor antagonist, increases dopaminergic transmission in the mesolimbic system. Neuropharmacology. 1999; 38(8):1195-1205.

41. DiMatteo V, DiGiovanni G, DiMascio M, Esposito E. Selective blockade of serotonin2C/2B receptors enhances dopamine release in the rat nucleus accumbens. Neuropharmacology. 1998;37(2):265-272.

42. Blier P, Szabo ST. Potential mechanisms of action of atypical antipsychotic medications in treatment-resistant depression and anxiety. J Clin Psychiatry. 2005;66 Suppl 8:30-40.

43. Seager MA, Huff KD, Barth VN, Phebus LA, Rasmussen K. Fluoxetine administration potentiates the effect of olanzapine on locus coeruleus neuronal activity. Biol Psychiatry. 2004;55(11):1103-1109.

44. Gobert A, Rivet JM, Lejeune F, et al. Serotonin(2C) receptors tonically suppress the activity of mesocortical dopaminergic and adrenergic, but not serotonergic, pathways: a combined dialysis and electrophysiological analysis in the rat. Synapse. 2000;36(3):205-221.

45. Ichikawa J, Ishii H, Bonaccorso S, Fowler WL, O'Laughlin IA, Meltzer HY. 5-HT(2A) and $\mathrm{D}(2)$ receptor blockade increases cortical DA release via 5-HT(1A) receptor activation: a possible mechanism of atypical antipsychotic-induced cortical dopamine release. J Neurochem. 2001;76(5):1521-1531.

46. Liegeois JF, Ichikawa J, Meltzer HY. 5-HT(2A) receptor antagonism potentiates haloperidol-induced dopamine release in rat medial prefrontal cortex and inhibits that in the nucleus accumbens in a dosedependent manner. Brain Res. 2002;947(2):157-165.

47. Ichikawa J, Meltzer HY. R(+)-8-OH-DPAT, a serotonin (1A) receptor agonist, potentiated $\mathrm{S}(-)$-sulpiride-induced dopamine release in rat medial prefrontal cortex and nucleus accumbens but not striatum. J Pharmacol Exp Ther. 1999;291(3):1227-1232. 
48. Jentsch JD, Roth RH, Taylor JR. Role for dopamine in the behavioral functions of the prefrontal corticostriatal system: implications for mental disorders and psychotropic drug action. Prog Brain Res. 2000;126: $433-453$.

49. Weisstaub NV, Zhou M, Lira A, et al. Cortical 5-HT2A receptor signaling modulates anxiety-like behaviors in mice. Science. 2006; 313(5786):536-540

50. Feiger AD, Heiser JF, Shrivastava RK, et al. Gepirone extended-release: new evidence for efficacy in the treatment of major depressive disorder. J Clin Psychiatry. 2003;64(3):243-249.

51. McGrath PJ, Stewart JW, Quitkin FM, et al. Gepirone treatment of atypical depression: preliminary evidence of serotonergic involvement. J Clin Psychopharmacol. 1994; 14(5):347-352.

52. Barde YA. Neurotrophins: a family of proteins supporting the survival of neurons. Prog Clin Biol Res. 1994;390:45-56.

53. Martinowich K, Manji H, Lu B. New insights into BDNF function in depression and anxiety. Nat Neurosci. 2007;10(9):1089-1093.

54. Duman RS, Monteggia LM. A neurotrophic model for stress-related mood disorders. Biol Psychiatry. 2006;59(12):1116-1127.

55. Sen S, Duman R, Sanacora G. Serum brain-derived neurotrophic factor, depression, and antidepressant medications: meta-analyses and implications. Biol Psychiatry. 2008;64(6):527-532.

56. Castren E, Voikar V, Rantamaki T. Role of neurotrophic factors in depression. Curr Opin Pharmacol. 2007;7(1):18-21.

57. Luo C, Xu H, Li XM. Post-stress changes in BDNF and Bcl-2 immunoreactivities in hippocampal neurons: effect of chronic administration of olanzapine. Brain Res. 2004;1025(1-2):194-202.

58. Shelton RC, Tollefson GD, Tohen M, et al. A novel augmentation strategy for treating resistant major depression. Am J Psychiatry. 2001;158(1):131-134.

59. Montgomery SA, Asberg M. A new depression scale designed to be sensitive to change. Br J Psychiatry. 1979;134:382-389.

60. Hamilton M. A rating scale for depression. J Neurol Neurosurg Psychiatry. 1960;23:56-62.

61. Clinical Global Impressions. ECDEU Assessment Manual for Psychopharmacology, Guy W, ed . 1976. Rockville, MD, U.S. Department of Health, Education, and Welfare, DHEW Publication No. (ADM) 76-338.

62. Shelton RC, Williamson DJ, Corya SA, et al. Olanzapine/fluoxetine combination for treatment-resistant depression: a controlled study of SSRI and nortriptyline resistance. J Clin Psychiatry. 2005; 66(10):1289-1297.

63. Corya SA, Williamson D, Sanger TM, Briggs SD, Case M, Tollefson G. A randomized, double-blind comparison of olanzapine/ fluoxetine combination, olanzapine, fluoxetine, and venlafaxine in treatment-resistant depression. Depress Anxiety. 2006;23(6): 364-372.

64. Thase ME, Corya SA, Osuntokun O, et al. A randomized, doubleblind comparison of olanzapine/fluoxetine combination, olanzapine, and fluoxetine in treatment-resistant major depressive disorder. J Clin Psychiatry. 2007;68(2):224-236.

65. Hamilton M. Hamiton Anxiety Scale. ECDEU Assessment Manual for Psychopharmacology, Guy W, ed, 193-198. 1976. Rockville, MD, U.S. Department of Health, Education, and Welfare.

66. Sheehan DV, Harnett-Sheehan K, Raj BA. The measurement of disability. Int Clin Psychopharmacol. 1996;11 Suppl 3:89-95.

67. Corya SA, Andersen SW, Detke HC, et al. Long-term antidepressant efficacy and safety of olanzapine/fluoxetine combination: a 76-week open-label study. J Clin Psychiatry. 2003;64(11):1349-1356.

68. Simpson GM, Angus JW. A rating scale for extrapyramidal side effects. Acta Psychiatr Scand Suppl. 1970;212:11-19.

69. Barnes TR. A rating scale for drug-induced akathisia. Br J Psychiatry. 1989;154:672-676.

70. Guy W. Abnormal Involuntary Movement Scale (AIMS). ECDEU Assessment Manual for Psychopharmacology, 534-537. 1976. Rockville, MD, National Institute for Mental Health.
71. Berman RM, Marcus RN, Swanink R, et al. The efficacy and safety of aripiprazole as adjunctive therapy in major depressive disorder: a multicenter, randomized, double-blind, placebo-controlled study. J Clin Psychiatry. 2007;68(6):843-853.

72. Marcus RN, McQuade RD, Carson WH et al. The efficacy and safety of aripiprazole as adjunctive therapy in major depressive disorder: a second multicenter, randomized, double-blind, placebo-controlled study. J Clin Psychopharmacol. 2008;28(2):156-165.

73. Thase ME. Quetiapine monotherapy for bipolar depression. Neuropsychiatr Dis Treat. 2008;4(1):11-21.

74. Daly EJ, Trivedi MH. A review of quetiapine in combination with antidepressant therapy in patients with depression. Neuropsychiatr Dis Treat. 2007;3(6):855-867.

75. Dodd S, Berk M. Olanzapine/fluoxetine combination for treatmentresistant depression: efficacy and clinical utility. Expert Rev Neurother. 2008;8(9):1299-1306.

76. Trivedi $\mathrm{MH}$, Thase ME, Osuntokun $\mathrm{O}$, et al. An integrated analysis of olanzapine/fluoxetine combination in clinical trials of treatmentresistant depression. J Clin Psychiatry. 2009;70(3):387-396.

77. Tohen M, Vieta E, Calabrese J, et al. Efficacy of olanzapine and olanzapine-fluoxetine combination in the treatment of bipolar I depression. Arch Gen Psychiatry. 2003;60(11):1079-1088.

78. Brown EB, McElroy SL, Keck PE Jr, et al. A 7-week, randomized, doubleblind trial of olanzapine/fluoxetine combination versus lamotrigine in the treatment of bipolar I depression. J Clin Psychiatry. 2006;67(7):1025-1033.

79. Brown E, Dunner DL, McElroy SL, et al. Olanzapine/fluoxetine combination vs lamotrigine in the 6-month treatment of bipolar I depression. Int J Neuropsychopharmacol. 2008 Dec 11:1-10. [Epub ahead of print]

80. Amsterdam JD, Shults J. Comparison of fluoxetine, olanzapine, and combined fluoxetine plus olanzapine initial therapy of bipolar type I and type II major depression - lack of manic induction. J Affect Disord. 2005;87(1):121-130.

81. Takahashi H, Kamata M, Yoshida K, Higuchi H, Ishigooka J. Augmentation with olanzapine in TCA-refractory depression with melancholic features: a consecutive case series. Hum Psychopharmacol. 2008;23(3):217-220.

82. Rasmussen K. Creating more effective antidepressants: clues from the clinic. Drug Discov Today. 2006;11(13-14):623-631.

83. Allison DB, Mentore JL, Heo M et al. Antipsychotic-induced weight gain: a comprehensive research synthesis. Am J Psychiatry. 1999;156(11):1686-1696.

84. American Diabetes Association, American Psychiatric Association, American Association of Clinical Endocrinologists, North American Association for the Study of Obesity. Consensus development conference on antipsychotic drugs and obesity and diabetes. Diabetes Care. 2004;27:596-601.

85. Newcomer JW. Second-generation (atypical) antipsychotics and metabolic effects: a comprehensive literature review. CNS Drugs. 2005;19 Suppl 1:1-93.

86. Iosifescu DV. Treating depression in the medically ill. Psychiatr Clin North Am. 2007;30(1):77-90.

87. Lett HS, Blumenthal JA, Babyak MA, et al. Depression as a risk factor for coronary artery disease: evidence, mechanisms, and treatment. Psychosom Med. 2004;66(3):305-315.

88. Davidson KW, Kupfer DJ, Bigger JT, et al. Assessment and treatment of depression in patients with cardiovascular disease: National Heart, Lung, and Blood Institute Working Group Report. Psychosom Med. 2006;68(5):645-650.

89. Frasure-Smith N, Lesperance F. Depression--a cardiac risk factor in search of a treatment. JAMA. 2003;289(23):3171-3173.

90. Hennekens $\mathrm{CH}$. Prevention of premature mortality among patients with schizophrenia: the need for primary prevention efforts in cardiovascular disease. CNS Spectr 2008; 13(6 Suppl 10):9-10.

91. Roshanaei-Moghaddam B, Katon W. Premature mortality from general medical illnesses among persons with bipolar disorder: a review. Psychiatr Serv. 2009;60(2):147-156. 
92. Berlim MT, Turecki G. What is the meaning of treatment resistant/ refractory major depression (TRD)? A systematic review of current randomized trials. Eur Neuropsychopharmacol. 2007;17(11):696-707.

93. Keller MB. Issues in treatment-resistant depression. J Clin Psychiatry. 2005;66 Suppl 8:5-12.

94. Rush AJ, Thase ME, Dube S. Research issues in the study of difficultto-treat depression. Biol Psychiatry 2003;53(8):743-753.
95. Sackeim HA. The definition and meaning of treatment-resistant depression. J Clin Psychiatry 2001;62 Suppl 16:10-17.

96. Trivedi MH, Morris DW, Grannemann BD, Mahadi S. Symptom clusters as predictors of late response to antidepressant treatment. J Clin Psychiatry. 2005;66(8):1064-1070.

\section{Publish your work in this journal}

Neuropsychiatric Disease and Treatment is an international, peerreviewed journal of clinical therapeutics and pharmacology focusing on concise rapid reporting of clinical or pre-clinical studies on a range of neuropsychiatric and neurological disorders. This journal is indexed on PubMed Central, the 'PsycINFO' database and CAS, and is the official

Submit your manuscript here: http://www.dovepress.com/neuropsychiatric-disease-and-treatment-journal journal of The International Neuropsychiatric Association (INA). The manuscript management system is completely online and includes a very quick and fair peer-review system, which is all easy to use. Visit http://www.dovepress.com/testimonials.php to read real quotes from published authors. 